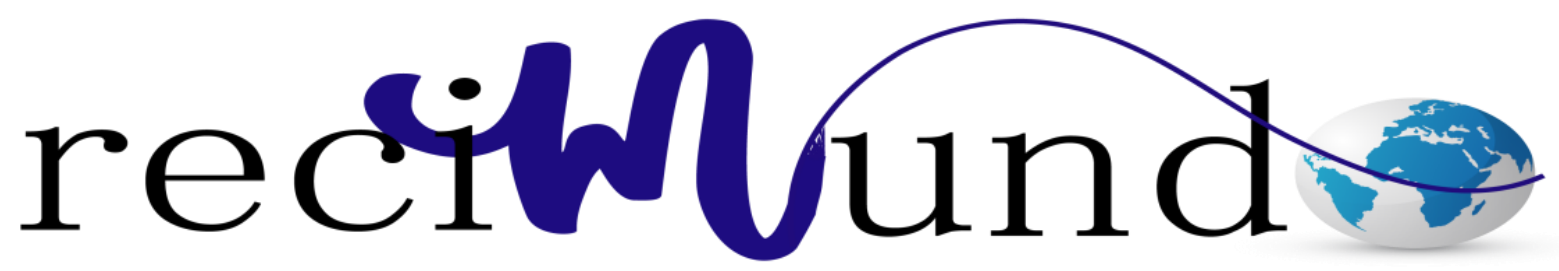

Revista Científica Mundo de la Investigación y el Conocimiento

Walter Manuel Camacho Ramírez ${ }^{\text {a }}$; Yuri Karolina Vera Castro ${ }^{\text {b }}$; Edwin Danilo Mendez Palomeque $^{\mathrm{c}}$

TIC: ¿Para qué? Funciones de las tecnologías de la información

TIC: For what? Functions of information technology

Revista Científica Mundo de la Investigación y el Conocimiento. Vol. 2 núm.3, julio, ISSN: 2588-073X, 2018, pp. 680-693

DOI: 10.26820/recimundo/2.(3).julio.2018.680-693

Editorial Saberes del Conocimiento

Recibido: 05/04/2018

Aceptado: 15/02/2018

Publicado: 30/07/2018

Correspondencia: walter.camacho@casagrande.edu.ec

a. Universidad Casa Grande; walter.camacho@casagrande.edu.ec

b. yurivera21@gmail.con

c. nilitom@gmail.com 


\section{TIC: ¿Para qué? Funciones de las tecnologías de la información}

Vol. 2, núm. 3., (2018)

Walter Manuel Camacho Ramírez; Yuri Karolina Vera Castro; Edwin Danilo Mendez

Palomeque

\section{RESUMEN}

En un mundo globalizado como el que hoy día nos encontramos, el conocimiento y empleo eficaz de las tecnologías que posibilitan el acceso a las diversas fuentes de información se ha convertido en una ventaja comparativa, mejor aún, cuando se logra conseguir información útil y confiable que permita ser aplicada a la vida cotidiana, profesional o en el contexto más conveniente, según el caso en particular. Es de allí que nace el interés por desarrollar este tema, relacionado con los usos y funciones de las Tecnologías de la Información y Comunicación (TIC) en un contexto fundamental. Esta idea se justifica en el hecho de que, si bien es cierto que mucho se ha escrito sobre este tema, no es menos cierto que últimamente estos se enfocan en la relación que tienen las TIC con un área en particular, sobre todo con la educación, administración, comunicación, entre otros, ocurriendo que, con la constante innovación y desarrollo de las tecnologías, se pierde sutilmente el amplio espectro que abarcan éstas, razón por la que este tema de las TIC sigue ampliándose a otras ramas en las que antes quizá no se les veía cabida. En este sentido, se puede decir que es de suma importancia refrescar su conceptualización y contextualización. Para alcanzar dicho objetivo, se ha propuesto llevar a cabo una investigación documental descriptiva, a fines de obtener resultados concretos, que no es más que la definición actualizada de las TIC, sus tipos, usos y funciones en general, quedando a criterio del lector aceptar o rechazar lo aquí expuesto.

Palabras clave: Tecnologías, información, innovación, contextualización, ventaja. 


\title{
TIC: ¿Para qué? Funciones de las tecnologías de la información
}

Vol. 2, núm. 3., (2018)

Walter Manuel Camacho Ramírez; Yuri Karolina Vera Castro; Edwin Danilo Mendez Palomeque

\begin{abstract}
In a globalized world like the one we find today, the knowledge and effective use of the technologies that allow access to diverse sources of information has become a comparative advantage, even better, when it is possible to obtain useful and reliable information. Allow to be applied to daily life, professional or in the most convenient context, depending on the particular case. It is from there that the interest to develop this topic, related to the uses and functions of the Information and Communication Technologies (ICT) in a fundamental context is born. This idea is justified in the fact that, although it is true that much has been written on this subject, it is no less true that lately these focus on the relationship that ICTs have with a particular area, especially with education., administration, communication, among others, occurring that, with the constant innovation and development of the technologies, the broad spectrum that these technologies are subtly lost, reason why this topic of the TIC continues expanding to other branches in which before perhaps they could not be accommodated. In this sense, it can be said that it is very important to refresh its conceptualization and contextualization. In order to achieve this objective, it has been proposed to carry out a descriptive documentary research, in order to obtain concrete results, which is nothing more than the updated definition of ICT, its types, uses and functions in general, leaving the reader to accept or reject what is stated here.
\end{abstract}

Key words: Technologies, information, innovation, contextualization, advantage. 


\section{TIC: ¿Para qué? Funciones de las tecnologías de la información}

Vol. 2, núm. 3., (2018)

Walter Manuel Camacho Ramírez; Yuri Karolina Vera Castro; Edwin Danilo Mendez

Palomeque

\section{Introducción.}

Es pertinente iniciar este tema haciendo referencia a Moreno (2015) quien afirma que:

Los artefactos tecnológicos tienen dos funciones principales: reproducir y producir. La tecnología digital, en sus distintos soportes, permite reproducir toda clase de mensajes y contenidos; pero, por fortuna, también permite crear o producir contenidos y desarrollar nuevas formas de representación y, lo que es mejor, nuestros propios contenidos. Así, una de las principales funciones de los medios tecnológicos o herramientas digitales (para el caso es lo mismo) es la de permitir su uso como medio de expresión y comunicación. (p.34)

Las tecnologías de la información y la comunicación (TIC) están referidas a la confluencia de las distintas herramientas tecnológicas que permiten producir, recibir, almacenar, compartir, acceder y procesar información, misma que es presentada a través de diferentes códigos (imágenes, textos, sonidos, entre otros). "El elemento más representativo en los últimos años es el ordenador y más recientemente, el uso de tecnologías portátiles como lo es el teléfono móvil (smartphone), entre otros.” (Hernández, 2017)

Estas tecnologías tienden a ocupar un lugar creciente en la vida humana y el funcionamiento de las sociedades debido a que se utilizan en multitud de actividades, y es por ello que han adoptado un papel importantísimo en nuestra sociedad en los últimos años. El uso de estas tecnologías no para de crecer y de extenderse, sobre todo en los países desarrollados, puesto que ya son parte significativa en la mayoría de los sectores, tales como educación, robótica, administración pública, empleo y empresas, militar, salud, entre otras, y en 


\section{TIC: ¿Para qué? Funciones de las tecnologías de la información}

Vol. 2, núm. 3., (2018)

Walter Manuel Camacho Ramírez; Yuri Karolina Vera Castro; Edwin Danilo Mendez Palomeque

retrospectiva, en los sociedades con menos avances tecnológicos, aumenta el riesgo de acentuar la brecha digital y social, y la diferencia entre generaciones.

En el mismo orden de ideas, es relevante destacar que la Comisión Económica para América Latina y el Caribe (CEPAL) se ha pronunciado respecto acerca de las tecnologías de la información, al afirmar, entre otras cosas, que:

Luego de más de una década de políticas para impulsar un mayor acceso y uso de estas tecnologías, los países de América Latina y el Caribe han logrado avances significativos en lo relativo al acceso a servicios de telecomunicaciones y al uso de aplicaciones y redes sociales, así como a la implementación de políticas y programas de educación, salud y gobierno electrónicos y la aprobación de los marcos regulatorios correspondientes. No obstante, avanzan a velocidades distintas y con grandes brechas, tanto entre ellos como en el interior de cada uno, además de con fuertes rezagos respecto de las economías más desarrolladas. (CEPAL, 2018)

\section{Materiales y Métodos.}

Para esta investigación se destinó el uso de un computador personal con acceso a internet como recurso fundamental para el desarrollo del tema, por medio del cual se pudo acceder a distintas fuentes de información digital ubicada en bases de datos nacionales y extranjeras, tanto de carácter formal como informal, sin embargo, con antelación se logró recabar una diversidad de material impreso relacionado con el tema propuesto, del que luego se hizo una selección, que respondía al criterio principal del presente trabajo investigativo, como lo es el uso y funciones de las TIC. 


\section{TIC: ¿Para qué? Funciones de las tecnologías de la información}

Vol. 2, núm. 3., (2018)

Walter Manuel Camacho Ramírez; Yuri Karolina Vera Castro; Edwin Danilo Mendez

Palomeque

De acuerdo a lo afirmado por (Ríos, 2017) respecto a la Tipología de las Investigaciones, se puede afirmar que el presente trabajo se enmarca dentro de la investigación documental, puesto a que "...recurre a la consulta de documentos para obtener sus resultados." y en cuanto a la profundidad que se pretende alcanzar, se puede decir que la misma es a nivel descriptivo, ya que “...busca encontrar las características, comportamiento y propiedades del objeto de estudio, ya sea en el presente o en el futuro...”(p.81).

Es importante destacar que el acceso a la información digital se efectuó a través del motor de búsqueda de Google Académico® y Google®. La búsqueda de dicha información se hizo de manera libre y aleatoria, teniendo como criterio las técnicas de información y comunicación, llegando a seleccionar del total del material disponible y consultado, sólo el contenido, que según criterio propio, resultó ser más adecuado y relevante.

\section{Resultados.}

Para Belloch (2015) las TIC "Son el conjunto de tecnologías que permiten el acceso, producción, tratamiento y comunicación de información presentada en diferentes códigos (texto, imagen, sonido)." Estas se desarrollan a partir de los avances científicos que se generan en áreas específicas como la informática y las telecomunicaciones" (p.1)

Así mismo, Díaz, Pérez, \& Florido (2011) recuren a la definición hecha por (Castañeda, 2003), quien afirma que las TIC son:

El resultado de las posibilidades creadas por la humanidad en torno a la digitalización de datos, productos, servicios y procesos y de su transportación a través de diferentes medios, a 


\section{TIC: ¿Para qué? Funciones de las tecnologías de la información}

Vol. 2, núm. 3., (2018)

Walter Manuel Camacho Ramírez; Yuri Karolina Vera Castro; Edwin Danilo Mendez Palomeque

grandes distancias y en pequeños intervalos de tiempo, de forma confiable, y con relación costobeneficio nunca antes alcanzadas por el hombre.(p.82)

Posteriormente los mismos autores agregan que en definitiva las TIC “...son el conjunto de procesos y productos derivados de las nuevas herramientas (hardware y software), soportes de la información y canales de comunicación relacionados con el almacenamiento, procesamiento y transmisión digitalizada de la información.” (p.82)

Características de las TIC

En la obra de Belloch (2015) se hace referencia a lo sintetizado de otros autores por Cabero (1998), quien al respecto, entre otra cosas, refiere que las TIC se caracterizan por:

- La inmaterialidad, porque las herramientas tecnológicas y recursos que comprenden las TIC, en líneas generales, efectúan la creación, el proceso y la comunicación de la información, razón por la que la misma puede ser considerada fundamentalmente inmaterial, condición por la que puede ser transmitida de forma inmediata y transparente a otros medios remotos.

- La interactividad, debido a la posibilidad de un intercambio de información entre el usuario y el ordenador, en otras palabras, se da la posibilidad de adaptar los recursos utilizados a las necesidades y características de los sujetos, en función de la interacción concreta del sujeto con el ordenador.

- La interconexión, por estar referencia a la creación de nuevas alternativas tecnológicas a partir de la conexión entre dos tecnologías. 


\section{TIC: ¿Para qué? Funciones de las tecnologías de la información}

Vol. 2, núm. 3., (2018)

Walter Manuel Camacho Ramírez; Yuri Karolina Vera Castro; Edwin Danilo Mendez

Palomeque

- La instantaneidad, porque las redes de comunicación y su integración con la informática, han posibilitado el uso de servicios que permiten la comunicación y transmisión de la información entre lugares remotos físicamente y de una forma rápida.

- La digitalización, su objetivo es que la información de distinto tipo (sonidos, texto, imágenes, animaciones, entre otros) pueda ser transmitida por los mismos medios al estar representada en un formato único universal.

- $\quad$ Altos niveles de calidad de imagen y sonido, el proceso y transmisión de la información abarca todo tipo de información: textual, imagen y sonido, por lo que los avances han ido encaminados a conseguir transmisiones multimedia de gran calidad, lo cual ha sido facilitado por el proceso de digitalización.

- Mayor Influencia sobre los procesos que sobre los productos, entendida como la posibilidad de que por el uso de diferentes aplicaciones de las TIC, éstas incidan positivamente sobre los procesos mentales que realizan los usuarios para la adquisición de conocimientos, más que sobre los propios conocimientos adquiridos. Esto se relaciona con los distintos análisis realizados sobre la sociedad de la información, en los que se remarca la enorme importancia de la inmensidad de información a la que permite acceder Internet. Sin embargo, también están los diversos autores que detractan esa teoría, argumentando justamente lo contrario, es decir, el efecto negativo de la proliferación de la información, los problemas de la calidad de la misma y la evolución hacia aspectos evidentemente sociales, pero menos ricos en potencialidad educativa, económica, comercial, lúdica, entre otras. No obstante, como otros tanto autores señalan, las posibilidades que brindan las TIC suponen un cambio cualitativo en los procesos más que en los 


\section{TIC: ¿Para qué? Funciones de las tecnologías de la información}

Vol. 2, núm. 3., (2018)

Walter Manuel Camacho Ramírez; Yuri Karolina Vera Castro; Edwin Danilo Mendez Palomeque

productos, ya que un sujeto no sólo dispone, a partir de las TIC, de una "masa" de información para construir su conocimiento sino que, además, puede construirlo en forma colectiva, asociándose a otros sujetos o grupos.

- $\quad$ Penetración en todos los sectores, esto es porque el impacto de las TIC no se refleja únicamente en un individuo, grupo, sector o país, sino que, se extiende al conjunto de las sociedades del planeta. Por esa razón, los efectos se extenderán a todos los habitantes, grupos e instituciones conllevando importantes cambios, cuya complejidad está en el debate social hoy en día.

- Innovación, porque las TIC constantemente están produciendo una innovación y cambio en todos los ámbitos sociales, no obstante, eso no debe entenderse como que por los cambios siempre se produce un rechazo a las tecnologías o medios anteriores, definitivamente no, puesto que en algunos casos se produce una especie de simbiosis con otros medios.

- Tendencia hacia automatización, la propia complejidad empuja a la aparición de diferentes posibilidades y herramientas que permiten un manejo automático de la información en diversas actividades personales, profesionales y sociales. La necesidad de disponer de información estructurada hace que se desarrollen gestores personales o corporativos con distintos fines y de acuerdo con unos determinados principios.

- Diversidad, la utilidad de las tecnologías puede ser muy diversa, desde la mera comunicación entre personas, hasta el proceso de la información para crear informaciones nuevas. 


\section{TIC: ¿Para qué? Funciones de las tecnologías de la información}

Vol. 2, núm. 3., (2018)

Walter Manuel Camacho Ramírez; Yuri Karolina Vera Castro; Edwin Danilo Mendez

Palomeque

Tipos de TIC

Según (Mela, 2011), las TIC generalmente se pueden clasificar de acuerdo a tres vertientes, a saber:

Redes.

Son las que conocemos como red telefonía fija, la banda ancha, la telefonía móvil, las redes de radio y televisión o las redes domésticas.

\section{Terminales.}

Son los dispositivos o terminales que forman parte de las TIC, tales como el ordenador (computadora), los teléfonos móviles, los televisores, los reproductores portátiles de audio y video, las consolas de juego, así como también lo son los sistemas operativos para ordenadores, el navegador de Internet, entre otros.

Servicios en las TIC.

Los más importantes son el correo electrónico, los motores de búsqueda, la banca online, el audio y música, la televisión y el cine, el comercio electrónico, e-administración y e-gobierno, la e-sanidad, la e-educación, los videojuegos y los servicios móviles, sin embargo, con el constante avance tecnológico, surgen nuevos servicios tales como los recientes P2P (Peer to Peer), los blogs o las comunidades virtuales y escuelas de negocio que se especializan en impartir su formación, entre otros. 


\section{TIC: ¿Para qué? Funciones de las tecnologías de la información}

Vol. 2, núm. 3., (2018)

Walter Manuel Camacho Ramírez; Yuri Karolina Vera Castro; Edwin Danilo Mendez Palomeque

De acuerdo con Hernández (2017) la gran cantidad de beneficios que pueden brindar las TIC dependerán ampliamente del uso que le demos y el valor que represente estas para el propio desarrollo. A pesar de ello, el autor menciona algunos beneficios generales que se desprenden del uso de las TIC, entre los que están:

- $\quad$ Facilitan la comunicación.

- $\quad$ Rompe las barreras de distancia, tiempo y espacio.

- $\quad$ Potencialmente, elevan la calidad de vida de las personas.

- $\quad$ En el proceso de aprendizaje, representan un recurso muy útil.

- Concibiendo la internet como herramienta estándar de comunicación, permite el acceso equitativo a la información y a la comunicación.

- $\quad$ El acceso a la información y divulgación científica es universal.

Limitantes para el desarrollo de las TIC

Díaz, Pérez, \& Florido (2011) refieren que la expansión de las TIC es un proceso continuo en todos los ámbitos de nuestra sociedad y que el mismo se ha producido aceleradamente, debido a que no se detiene el surgimiento de nuevas tecnologías y aunque estos precedentes ocasionen que las TIC sean valoradas como medios bastamente útiles en cualquier contexto, existen múltiples situaciones que truncan de cierta manera su usabilidad entre las actividades y capas sociales, es decir, que ocasionan que las TIC no funcionen correctamente, y aunque parezca contradictorio, tras la evolución de las mismas se contribuye a marcar la brecha 


\section{TIC: ¿Para qué? Funciones de las tecnologías de la información}

Vol. 2, núm. 3., (2018)

Walter Manuel Camacho Ramírez; Yuri Karolina Vera Castro; Edwin Danilo Mendez

Palomeque

digital cada día más, basada fundamentalmente en aspectos de acceso, pero adicional están los relacionados con el uso propiamente de las TIC.

En relación a la "brecha digital" los autores agregan que, por mencionar una de sus causales, se da cuando a algunos sectores o grupos se les imposibilita acceder a tales medios y recursos tecnológicos, y como consecuencia ocasiona que dichos grupos no obtengan las mismas oportunidades de acceso a la información y al conocimiento, tal y como ocurre en los países no desarrollados en comparación con los que sí lo son.

\section{Conclusiones.}

Se considera de suma importancia concluir haciendo referencia a algunas ideas encontradas en un documento de proyecto ejecutado por la División de Desarrollo Productivo y Empresarial de la CEPAL, titulado "Estrategias de TIC ante el desafío del cambio estructural en América Latina y el Caribe. Balance y retos de renovación", no tanto porque este trabajo investigativo este contextualizado en nuestra zona geográfica, sino porque en líneas generales aporta las ideas que se pretenden transmitir con esta investigación, que no sería más que los distintos usos y funciones que poseen las TIC, idea que desde la introducción de esta investigación se quiere dejar clara. Lo expuesto en ese documento es lo siguiente:

Las TIC, como tecnologías de propósito general, pueden contribuir a modernizar y revitalizar las actividades productivas tradicionales de la región, así como a tornar más eficientes e inclusivos los servicios públicos. Las nuevas plataformas y redes de información están transformando radicalmente los sistemas de toma de decisiones y modelos operativos en industrias como la minería, la pesca, la agricultura, el transporte y el turismo, así como en 


\section{TIC: ¿Para qué? Funciones de las tecnologías de la información}

Vol. 2, núm. 3., (2018)

Walter Manuel Camacho Ramírez; Yuri Karolina Vera Castro; Edwin Danilo Mendez Palomeque

muchos otros servicios. Los Datos Abiertos y el Big Data están llamados a revolucionar completamente la gestión de información, seguridad y logística en las grandes ciudades (smart cities). Las pequeñas empresas pueden apoyarse en las TIC para dar saltos en la eficiencia de su gestión y en sus sistemas productivos y comerciales, así como para ingresar en mercados antes difíciles de alcanzar, como el de compras públicas y el internacional. El comercio electrónico abre nuevas oportunidades para las micro y pequeñas empresas que consigan participar de estas plataformas. La región también puede aprovechar toda esa nueva demanda por bienes y servicios TIC que crece día a día en los sectores públicos y privados para apalancar la consolidación y surgimiento de nuevas empresas y sectores TIC capaces de proveer equipos, soluciones $\mathrm{y}$ servicios para esos mercados en expansión, incluso con posibilidades de insertarse en cadenas internacionales de valor (outsourcing). (CEPAL, 2013) (p.9)

\section{Referencias Bibliográficas}

Barranco, R. (2012). ¿Qué es Big Data? Todos formamos parte de ese gran crecimiento de datos. Recuperado de: https://www.ibm.com/developerworks/ssa/local/im/que-es-big-data/index.html

Belloch, C. (2015). www.uv.es. Obtenido de www.uv.es: https://www.uv.es/ bellochc/pdf/pwtic1.pdf

CEPAL. (03 de 2013). www.cepal.org. Obtenido de www.cepal.org: https://repositorio.cepal.org/bitstream/handle/11362/4063/S2013159_es.pdf?sequence=1\&isAllo wed $=\mathrm{y}$

CEPAL. (2012). www.cepal.org. Obtenido de www.cepal.org: https://repositorio.cepal.org/bitstream/handle/11362/7331/1/S1200084_es.pdf

CEPAL. (2018). www.cepal.org. Obtenido de www.cepal.org: https://www.cepal.org/es/temas/tecnologias-de-la-informacion-y-las-comunicaciones-tic/acercatecnologias-la-informacion 


\section{TIC: ¿Para qué? Funciones de las tecnologías de la información}

Vol. 2, núm. 3., (2018)

Walter Manuel Camacho Ramírez; Yuri Karolina Vera Castro; Edwin Danilo Mendez

Palomeque

Díaz, J., Pérez, A., \& Florido, R. (2011). Impacto de las Tecnologías de la Información y las Comunicaciones (TIC) para Disminuir la Brecha Digital en la Sociedad Actual. Cultura Tropical, 32(1), 81-90. Obtenido de Cultivos Tropicales, 32(1), 8190.http://scielo.sld.cu/scielo.php?script=sci_arttext\&pid=S0258-

59362011000100009\&lng=es\&tlng=es.

Hernández, E. (17 de 03 de 2017). www.lainter.edu.mx. Obtenido de www.lainter.edu.mx: https://www.lainter.edu.mx/blog/2017/03/17/las-tics-eliminan-la-barrera-de-distancia-tiempo-yespacio/

Mela, M. (13 de 04 de 2011). http://www.iberestudios.com/. Obtenido de http://www.iberestudios.com/: http://noticias.iberestudios.com/\%C2\%BFque-son-las-tic-y-paraque-sirven/

Moreno, I. (2015). www.academia.edu. Obtenido de /www.academia.edu: http://www.academia.edu/20313021/_Para_qu\%C3\%A9_sirven_las_TIC

Ríos, R. (09 de 2017). www.eumed.net. (E. Grupo de investigación (SEJ 309) eumed.net de la Universidad de Málaga, Ed.) Obtenido de www.eumed.net: http://www.eumed.net/libros/libro.php?id=1662 